UMR 5824

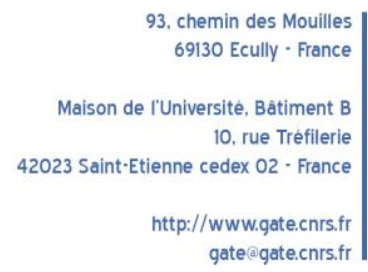

WP 1717 - June 2017

\title{
Collaborateurs, emplois familiaux et niveau d'activité des parlementaires français
}

\author{
Benjamin Monnery
}

\begin{abstract}
:
Cet article profite de la récente publication, à la suite de l' "affaire Fillon", d'une liste exhaustive des collaborateurs employés par l'ensemble des 920 parlementaires français actuels, pour mesurer l'emploi de membres de la famille par les députés et sénateurs. En rapprochant ces informations aux données démographiques et politiques des parlementaires, on en tire quelques enseignements sur le profil-type des élus ayant recours à des emplois familiaux. Enfin, en croisant ces statistiques avec les données publiques disponibles sur l'activité des parlementaires observée sur les 12 derniers mois, on montre que les parlementaires qui emploient comme collaborateurs des membres de leur famille sont en moyenne significativement moins présents, moins actifs et moins productifs à l'Assemblée et au Sénat que les autres, toutes choses égales par ailleurs. Ce résultat suggère soit que l'emploi de collaborateurs familiaux (en substitution d'autres collaborateurs) réduit l'activité du parlementaire (effet causal), soit que les parlementaires qui emploient un membre de leur famille sont par ailleurs des élus relativement peu actifs au Parlement (effet de sélection). Des estimations par variable instrumentale semblent plutôt privilégier la deuxième hypothèse.
\end{abstract}

\section{Keywords:}

Economie politique, extraction de rente, népotisme, Parlement

\section{JEL codes:}

D72 


\title{
Collaborateurs, emplois familiaux et niveau d'activité des parlementaires français
}

\author{
Benjamin Monneryin \\ GATE CNRS, Université Lyon 2
}

Juin 2017

\section{Résumé}

Cet article profite de la récente publication, à la suite de l' "affaire Fillon", d'une liste exhaustive des collaborateurs employés par l'ensemble des 920 parlementaires français actuels, pour mesurer l'emploi de membres de la famille par les députés et sénateurs. En rapprochant ces informations aux données démographiques et politiques des parlementaires, on en tire quelques enseignements sur le profil-type des élus ayant recours à des emplois familiaux. Enfin, en croisant ces statistiques avec les données publiques disponibles sur l'activité des parlementaires observée sur les 12 derniers mois, on montre que les parlementaires qui emploient comme collaborateurs des membres de leur famille sont en moyenne significativement moins présents, moins actifs et moins productifs à l'Assemblée et au Sénat que les autres, toutes choses égales par ailleurs. Ce résultat suggère soit que l'emploi de collaborateurs familiaux (en substitution d'autres collaborateurs) réduit l'activité du parlementaire (effet causal), soit que les parlementaires qui emploient un membre de leur famille sont par ailleurs des élus relativement peu actifs au Parlement (effet de sélection). Des estimations par variable instrumentale semblent plutôt privilégier la deuxième hypothèse.

Mots-clés : political economy, rent-extraction, nepotism, Parliament

JEL : D72

$\dagger$ Univ Lyon, Université Lumière Lyon 2, GATE L-SE UMR 5824, F-69130 Ecully, France. Email : monnery@gate.cnrs.fr

Je remercie chaleureusement les membres de l'association Regards Citoyens pour leur travail de retranscription des listes des collaborateurs (PDF) en données ouvertes exploitables sous licence ODbL, et pour la diffusion rapide de ces données en ligne. Je remercie aussi les Décodeurs du Monde pour les données issues de l'opération \#TransparenceAN. Ce travail a bénéficié de commentaires utiles de la part de François Briatte, Ewen Gallic, Jean-Yves Lesueur, Rémi Suchon et François-Charles Wolff. 


\begin{abstract}
This article takes advantage of the recent release, following the "Fillon scandal", of a list of all assistants employed by the 920 current parliamentarians in France, to quantify family employment by deputies and senators. By linking this information with demographic and political characteristics, we give a portray of parliamentarians who employ family members. Finally, after merging these statistics to publicly-available data on officials' observable activity in the Parliament during the last 12 months, we show that parliamentarians who employ family members as assistants are significantly less present, less active and less productive in the Assembly and in the Senate compared to others, all else equal. This result either suggests that employing a family member (instead of other assistants) reduces activity in the Parliament (causal effect), or that those who use family jobs are also relatively low-activity parliamentarians (selection effect). Instrumental variable regressions tend to favor the second hypothesis.
\end{abstract}




\section{Introduction}

Le travail des parlementaires et de leurs collaborateurs est au coeur de l'actualité politique française, depuis la révélation de 1' "affaire Fillon" par Le Canard Enchainé en Janvier 2017. François Fillon a en effet employé et rémunéré pendant plusieurs années son épouse comme collaboratrice, ainsi que deux de ses enfants, sur son "crédit parlementaire" (enveloppe budgétaire consacrée à l'emploi de collaborateurs, de 9561 euros par mois à l'Assemblée Nationale, et de 7500 euros au Sénat ${ }^{17}$. Comme le suggèrent l'enquête du journal et les investigations qui ont suivi, ces emplois familiaux pourraient se révéler fictifs, la réalité du travail effectué paraissant difficile à établir. Ces élements ont conduit le Parquet National Financier à mettre le couple Fillon en examen pour détournement de fonds publics et abus de biens sociaux. Depuis le déclenchement de cette affaire, la pratique des emplois familiaux chez les parlementaires est devenu un sujet de débat public, et un projet de loi (dit "de moralisation de la vie politique") prévoit d'interdire cette pratique en France.

Du point de vue de la théorie économique, l'emploi d'un proche sur fonds publics peut se modéliser comme une extraction de rentes par un agent (le député) dont le comportement est difficilement observable et sanctionnable par le principal (les électeurs). Dans sa contribution séminale, Barro (1973) montre comment diverses caractéristiques des institutions politiques peuvent nuire à l'efficacité du contrôle citoyen que constituent les élections, et générer des opportunités d'extraction de rentes pour les élus. De manière très stylisée, l'emploi d'un membre de la famille par les parlementaires est cohérent avec un comportement de maximisation d'une fonction d'utilité de type : $U=f\left(W, W_{f}, e\right)$. L'utilité de l'élu dépend positivement de son revenu $(W)$ et de celui de sa famille $\left(W_{f}\right)$, et négativement de son effort au Parlement $(e)$. Ce type de parlementaire opportuniste a tout intérêt à employer un proche, et à fournir un effort faible. Depuis Barro (1973), plusieurs résultats empiriques suggèrent que les carrières politiques peuvent être avantageuses financièrement : obtenir un siège de parlementaire en Grande Bretagne améliore par exemple fortement les chances d'obtenir un poste de direction dans une entreprise côtée plus tard (Eggers et Hainmueller, 2009). En Italie, Gagliarducci et Manacorda (2016) montrent que les membres de la famille des élus bénéficient professionnellement de ce mandat, avec une plus forte probabilité d'avoir un emploi dans le secteur privé après l'élection. D'autres travaux confirment également le caractère opportuniste de divers comportements politiques, comme les changements de parti au parlement turc (Altindag et Mocan, 2015), la poursuite

\footnotetext{
${ }^{1}$ Les parlementaires sont totalement libres du choix de leurs collaborateurs, dans la limite de 5 maximum. Les salaires de leurs proches sont cependant encadrés depuis 1997 : à l'Assemblée, les proches ne peuvent recevoir plus de la moitié de l'enveloppe totale. Au Sénat, les époux-ses peuvent toucher au maximum un tiers de l'enveloppe (soit 2500 euros), et les autres proches jusqu'à 3800 euros.
} 
de carrières privées au détriment du mandat public en Italie (Gagliarducci et al., 2010), ou l'embauche de membres de la famille en Allemagne. Sur ce dernier point, Kauder et Potrafke (2016) montrent qu'en Bavière, les parlementaires ayant les meilleures chances de réélection sont plus susceptibles d'embaucher un membre de leur famille sur fonds publics que les parlementaires dont la réélection est plus incertaine. Ces résultats suggèrent que les députés bavarois considèrent cette pratique comme profitable individuellement mais politiquement risquée. Si l'emploi familial relève d'une stratégie d'extraction de rentes, comme l'interprêtent Kauder et Potrafke (2016), on s'attend donc à ce que cette pratique soit corrélée à d'autres comportements opportunistes, comme une moindre présence au Parlement et une participation réduite aux travaux parlementaires (interventions orales, amendements, questions au gouvernement...).

Une autre approche consiste à appréhender le travail parlementaire comme l'output d'une fonction de production, dans laquelle les collaborateurs jouent un rôle d'input. Navarro et al. (2012) et François et Weill (2014) appliquent cette approche sur des données françaises pour évaluer l'efficacité du travail des députés par des méthodes de frontières non-paramétriques (mise en balance des activités réalisées et du temps de travail consacré). Dans le cas des emplois familiaux, la fonction de production parlementaire peut s'écrire sous la forme : $Y=f\left(L_{f}, L_{n f}, e\right)$. La production observable du parlementaire, $Y$, dépend alors du travail fourni par ses collaborateurs, qu'ils soient membres de la famille $\left(L_{f}\right)$ ou non $\left(L_{n f}\right)$, et d'autres caractéristiques de l'élu telles que son effort ou sa compétence $(e)$. Cette approche suppose un lien de causalité entre l'emploi familial et la production parlementaire. Ce lien peut être négatif $\left(Y^{\prime}\left(L_{f}\right) \leq 0\right)$, si l'emploi familial est en réalité un emploi fictif ou si l'embauche d'un proche se fait au détriment d'un autre candidat plus compétent (par exemple du fait de l'existence d'une contrainte budgétaire²). A l'inverse, certains parlementaires expliquent que l'emploi de membres de la famille permet une plus grande efficacité, grâce à leur proximité, leur confiance mutuelle, et les horaires de travail plus flexibles dans ce type de relations ${ }^{3}$. Sous cette hypothèse, on s'attend à un effet causal positif de l'emploi familial sur la production parlementaire.

Dans la suite de cet article, nous estimons la relation empirique entre nombre de collaborateurs, emploi familial et activités parlementaires. Auparavant, la section 2 présente quelques statistiques sur les collaborateurs des 920 parlementaires français actuels, et quantifie les emplois familiaux en procédant par un appariemment sur les noms de famille (une méthode simple mais qui se révèle relativement fiable). La section 3 dresse un portrait-type des parlementaires

\footnotetext{
${ }^{2}$ Dans la pratique, les parlementaires sont bien soumis à une contrainte budgétaire, le "crédit parlementaire", de type $w_{f} L_{f}+w_{n f} L_{n f} \leq R$, si bien que le nombre de postes de collaborateurs est limité.

${ }^{3}$ Plusieurs députés ont fourni ce type d'explications à différents médias, par exemple à France Info http://www.francetvinfo.fr/politique/francois-fillon/penelope-fillon/ femmes-mari-ou-fille-de-depute-ils-sont-collaborateurs-parlementaires-ils-racontent-ou-pas-ce-qu-ils-font_ 2038765.html
} 
employant des membres de leur famille, en fonction de quelques caractéristiques démographiques et politiques. Enfin, la section 4 procède aux estimations économétriques.

\section{Etat des lieux de l'emploi familial à l'Assemblée et au Sénat}

Le scandale provoqué par l'affaire Fillon a poussé l'Assemblée Nationale et le Sénat à publier sur leurs sites web, le 21 février 2017, les listes nominatives des collaborateurs des 920 députés et sénateurs actuels. L'association Regards Citoyens a re-publié le lendemain ces listes dans un format ouvert exploitable (fichiers .csv), ce qui permet d'estimer la quantité d'emplois familiaux actuels au Parlement. En comparant les noms de famille (éventuellement noms composés) des 920 parlementaires et de leurs 3027 collaborateurs déclarés, on identifie 129 parlementaires $(14,0 \%)$ partageant le même nom de famille qu'au moins un de leurs collaborateurs. Parmi ceux-ci, 86 sont députés et représentent 15,0\% de l'effectif de l'Assemblée, et 43 sont sénateurs (12,4\% du Sénat).

Si les soupçons d'emploi fictif sont propres à l'affaire Fillon, la question des emplois familiaux au Parlement français n'est pas nouvelle. Dès 2014, après l'affaire Cahuzac et la mise en place de la Haute Autorité pour la Transparence de la Vie Publique (HATVP), tous les parlementaires avaient dû déclarer par écrit leurs intérêts ainsi que la liste de leurs collaborateurs parlementaires. En cas de déclaration fausse ou incomplète, les parlementaires encourraient des sanctions pénales. Les informations déclarées, rendues publiques en juillet 2014, avaient permis à Mediapart d'enquêter en détail sur les emplois familiaux à l'Assemblée Nationale : leur enquête révélait qu'au moins 115 députés (soit 20\%) employaient ou avaient employé, en CDD ou CDI, au moins un membre de leur famille: 52 époux(-ses), 28 fils, 32 filles, 2 cousins(-ines), et 1 petite-fille de député. Cet article de Mediapart est le fruit d'un long travail d'enquête, mêlant analyse des noms de famille des députés et de leurs collaborateurs et enquête de terrain (pour confirmer l'analyse par patronyme et identifier d'autres cas d'emplois familiaux antérieurs à la déclaration d'intérêts).

Sur ces 115 députés identifiés par ce travail qualitatif de Mediapart, on pouvait cependant à l'époque en détecter 89 (soit 77\%) par la méthode de comparaison des noms de famille des parlementaires et de leurs collaborateurs. Ces chiffres suggèrent que cette méthode d'appariement est un indicateur fiable, quoique conservateur, de la réalité de l'emploi familial à l'Assemblée. Ils suggèrent aussi que la situation a peu évolué entre juillet 2014 et février 2017, le nombre de députés usant d'emplois familiaux passant de 89 à 86 députés. Enfin, la même méthode appliquée aux déclarations d'intérêts du Sénat de 2014 identifie 36 sénateurs employant au moins 
Table 1: Emplois familiaux par député en février 2017 : estimations contre déclarations

Données de l'opération \#TransparenceAN

Par appariemment

\begin{tabular}{lcccc} 
sur noms de famille & 0 & 1 & 2 & Total \\
\hline 0 & 189 & 20 & 1 & 210 \\
1 & 0 & 76 & 3 & 79 \\
2 & 0 & 0 & 5 & 5 \\
\hline Total & 189 & 96 & 9 & 294 \\
\hline
\end{tabular}

un membre de leur famille. Là encore, la situation a donc peu évolué entre 2014 et 2017, passant de 36 à 43 sénateurs.

Une autre méthode permettant de vérifier la pertinence des estimations de l'emploi familial obtenues consiste à exploiter les déclarations volontaires fournies par les députés eux-mêmes en février 2017 lors de l'opération \#TransparenceAN. Cette opération, lancée par différents médias et acteurs de la société civile au coeur de l'affaire Fillon, avait pour but de rendre les pratiques de l'Assemblée Nationale plus transparentes, en demandant notamment à tous les députés de déclarer s'ils comptaient des collaborateurs familiaux dans leur équipe parlementaire. Le journal Le Monde a ainsi recueilli les réponses de 223 députés sur 572. En complétant ces déclarations volontaires par d'autres informations publiques (presse, sources officielles), le journal a recueilli des données fiables et à jour sur 294 députés. Le nombre d'emplois familiaux identifiés par $L e$ Monde correspond pour $92 \%$ des députés (270 sur 294) à celui obtenu par simple appariemment sur les noms de famille. Comme le montre la Table 1, la méthode par appariemment identifie 84 situations d'emploi familial, alors qu'on en dénombre en réalité 105 selon les données issues de l'opération \#TransparenceAN, soit un taux de détection de $80 \%$.

\section{Profil des parlementaires employant des membres de leur famille}

La Table 2 donne des statistiques descriptives sur le nombre total de collaborateurs déclarés par les parlementaires. Le parlementaire moyen emploie 3,3 collaborateurs (médiane de 3). En moyenne, les députés ont sensiblement plus de collaborateurs $(3,7)$ que les sénateurs $(2,7)$, probablement car l'enveloppe budgétaire disponible pour les sénateurs est plus limitée. Il n'existe pas de différences nettes entre parlementaires hommes et parlementaires femmes, ni en fonction de l'âge ou du nombre de mandats actuels des parlementaires.

En revanche, les statistiques reportées dans la Table 3 font apparaitre de fortes différences entre parlementaires dans l'emploi de membres de leur famille. Tout d'abord, rappelons que 129 des 920 parlementaires actuels (14,0\%) emploient un membre de leur famille (selon la 
méthode d'appariement par noms de famille). L'emploi familial est légèrement plus présent chez les députés $(15 \%)$ que chez les sénateurs $(12,4 \%)$. Surtout, cette pratique est beaucoup plus courante parmi les parlementaires hommes que parmi les femmes $(15,7 \%$ et $9,4 \%)$ et parmi les parlementaires les plus âgés (17,8\% chez les plus de 62 ans, l'age médian actuellement, et $10,2 \%$ chez les moins de 62 ans). Enfin, l'emploi familial semble plus courant chez les parlementaires cumulant plusieurs mandats $(14,7 \%)$ que chez les parlementaires exerçant un seul mandat $(9,5 \%)$.

Table 2: Nombre de collaborateurs par parlementaire au 21 février 2017

\begin{tabular}{lccccc}
\hline \hline NbCollab & Moyenne & Médiane & Min & Max & N \\
\hline $\begin{array}{l}\text { Tous } \\
\text { Par chambre : }\end{array}$ & 3.29 & 3 & 1 & 8 & 920 \\
$\quad$ & & & & & \\
$\quad$ Députés & 3.66 & 4 & 1 & 8 & 572 \\
$\quad$ Sénateurs & 2.68 & 3 & 1 & 5 & 348 \\
Par genre : & 3.29 & 3 & 1 & 8 & 676 \\
$\quad$ Hommes & 3.30 & 3 & 1 & 7 & 244 \\
$\quad$ Femmes & 3.21 & 3 & 1 & 7 & 467 \\
Par age (+/- 62 ans) : & 3.37 & 3 & 1 & 8 & 453 \\
$\quad+62$ ans & & & & & \\
$\quad$ - 62 ans & 3.28 & 3 & 1 & 7 & 116 \\
Par nombre de mandats : & 3.29 & 3 & 1 & 8 & 804 \\
$\quad$ Un seul mandat & & & & & \\
$\quad$ Plusieurs mandats & & & & & \\
\hline
\end{tabular}

La Table 5 renseigne enfin les différences dans l'emploi familial entre groupes parlementaires. Ces différences ne sont pas statistiquement significatives (probablement du fait du faible nombre de parlementaires dans certains groupes), mais révèlent tout de même des écarts notables. Le groupe écologiste au Sénat n'emploie ainsi aucun collaborateur familial, contre plus de $20 \%$ parmi les députés Les Républicains et Non-Inscrits.

\section{Nombre de collaborateurs, emplois familiaux, et niveau d'activité des par- lementaires}

\subsection{Méthode}

Pour étudier le lien entre le nombre de collaborateurs, l'emploi de membres de la famille, et le niveau d'activité observé des parlementaires, nous passons à une analyse économétrique à l'aide des données disponibles sur l'activité totale des parlementaires sur les 12 derniers mois (depuis 
Table 3: Collaborateurs familiaux au 21 février 2017

\begin{tabular}{lcccc}
\hline \hline NbFamily & 0 & 1 & 2 & $\mathrm{~N}$ \\
\hline $\begin{array}{l}\text { Tous } \\
\text { Par chambre : }\end{array}$ & 791 & 124 & 5 & 920 \\
$\quad$ Députés & 486 & 81 & 5 & 572 \\
$\quad$ Sénateurs & 305 & 43 & 0 & 348 \\
Par genre : & & & & \\
$\quad$ Hommes & 570 & 101 & 5 & 676 \\
$\quad$ Femmes & 221 & 23 & 0 & 244 \\
Par age (+/- 62 ans) : & & & & \\
$\quad+62$ ans & 384 & 78 & 5 & 467 \\
$\quad$ - 62 ans & 407 & 46 & 0 & 453 \\
Par nombre de mandats : & & 11 & & \\
$\quad$ Un seul mandat & 105 & 113 & 5 & 804 \\
$\quad$ Plusieurs mandats & 686 & & & \\
\hline
\end{tabular}

Table 4: Part des parlementaires employant un membre de leur famille

\begin{tabular}{|l|cc|c|}
\hline Profil & -60 ans & +60 ans & Tous \\
\hline Femmes & $6,50 \%$ & $12,40 \%$ & $9,43 \%$ \\
Hommes & $9,45 \%$ & $19,95 \%$ & $15,68 \%$ \\
\hline Tous & $8,54 \%$ & $18,20 \%$ & $14,02 \%$ \\
\hline
\end{tabular}

Table 5: Part des parlementaires employant un membre de leur famille, par groupe parlementaire

\begin{tabular}{lcc}
\hline \hline Groupe parlementaire & Assemblée Nationale & Sénat \\
\hline GDR $(\mathrm{N}=15)$ & $6,67 \%$ & - \\
SER $(\mathrm{N}=288)$ & $9,38 \%$ & - \\
RRDP $(\mathrm{N}=18)$ & $16,67 \%$ & - \\
CRC $(\mathrm{N}=21)$ & - & $9,52 \%$ \\
RDSE $(\mathrm{N}=17)$ & - & $17,65 \%$ \\
ECO $(\mathrm{N}=10)$ & - & $0,00 \%$ \\
SOC $(\mathrm{N}=108)$ & - & $12,04 \%$ \\
UDI $(\mathrm{N}=27+42)$ & $18,52 \%$ & $14,29 \%$ \\
LR $(\mathrm{N}=199+144)$ & $22,11 \%$ & $12,50 \%$ \\
NI $(\mathrm{N}=25+6)$ & $24,00 \%$ & $16,67 \%$ \\
\hline Tous & $14,34 \%$ & $11,78 \%$ \\
\hline
\end{tabular}


février 2016), compilées par Regards Citoyens sur les sites nosdeputes.fr et nossenateurs.fr. Ces données recensent, pour chacun des 920 parlementaires actuels, le nombre total de leurs activités sur un an mesurables à partir des informations publiques de l'Assemblée, du Sénat, et du Journal Officiel. Nous excluons de la suite de l'analyse les 24 parlementaires ayant commencé leur mandat il y a moins de 12 mois, puisque les variables d'activités sont mesurées sur un an. L'échantillon final comprend donc 896 parlementaires. Les activités mesurées sont les suivantes :

1. Semaines : nombre total de semaines où le parlementaire est détecté présent

2. ComPres : nombre total de présences en réunions de commissions

3. ComInterv : nombre total d'interventions orales en commissions

4. HemiInterv : nombre total d'interventions orales dans l'hémicycle

5. Rapports : nombre total de rapports parlementaires rendus

6. AmenSign : nombre total d'amendements signés

7. AmenAdo : nombre total d'amendements adoptés

8. Ado/Sign : ratio du nombre d'amendements adoptés par amendements signés

9. PropEcrit : nombre total de propositions de lois écrites

10. PropSign : nombre total de propositions de lois signées

11. QEcrites : nombre total de questions écrites au gouvernement

12. QOrales : nombre total de questions orales au gouvernement

Compte tenu du grand nombre de variables d'activité disponibles, nous tentons dans un premier temps de les synthétiser en un unique score d'activité. Pour cela, nous recourons à une Analyse en Composante Principale dans laquelle sont inclues les 12 variables en question (avec les variables 3 à 12 calculées sous forme logarithmique pour tenir compte de leur forte dispersion à droite ${ }^{4}$. Le premier facteur (ou composante princpale) issu de l'ACP permet la construction d'un score individuel d'activité qui sert de variable dépendante dans l'analyse économétrique.

Cependant, ce premier facteur ne capte qu'une partie de la corrélation entre les différents indicateurs d'activité (contribution de 31\%), ce qui témoigne du caractère multi-dimensionnel du travail des parlementaires. Pour rendre compte de la richesse de ces activités, et de leur lien

\footnotetext{
${ }^{4}$ Pour mesurer la forte dispersion à droite de certaines variables d'activité, la Figure 1 en Annexe fournit des histogrammes.
} 
avec les collaborateurs familiaux, nous utilisons aussi les 12 indicateurs d'activité séparément, comme variables dépendantes de nos régressions économétriques.

Ces régressions sont de la forme :

$$
\text { Activite }_{i}=\beta_{0}+\beta_{1} \text { EmploiFamilial }_{i}+\beta_{2} \text { NbCollab }_{i}+\theta^{\prime} X_{i}+u_{i}
$$

où Activite correspond soit au score d'activité global issu de l'ACP, soit à l'un des 12 indicateurs d'activité mesuré en niveau (Semaines, ComPres) ou en logarithme (les 10 autres indicateurs) $\sqrt[5]{5}$ sur les 12 derniers mois (février 2016-février 2017), EmploiFamilial est une indicatrice valant 1 si le parlementaire emploie au moins un membre de sa famille et 0 sinon, NbCollab est une variable continue mesurant le nombre total de collaborateurs déclarés par le parlementaire, et $X$ est un vecteur de caractéristiques démographiques et politiques pouvant expliquer l'activité des parlementaires ainsi que l'emploi familial. Dans la spécification de base du modèle, ce vecteur comprend le genre, l'age, la chambre (Assemblée ou Sénat), le fait de cumuler ou non plusieurs mandats, et le fait d'être élu des DOM-TOM ou des Français de l'étranger. Ce vecteur de contrôle peut aisément être enrichi, par exemple pour tenir compte du groupe parlementaire, de la longévité politique du parlementaire ou même du département d'élection (résultats similaires).

Le principal coefficient d'intérêt dans cette régression est $\beta_{1}$, qui mesure les différences moyennes d'activité entre les parlementaires qui emploient un membre de leur famille et ceux qui n'en emploient pas, toutes choses égales par ailleurs. Ce coefficient $\beta_{1}$ devrait être positif si le fait d'employer un membre de sa famille plutôt qu'un autre collaborateur est associé à un meilleur niveau d'activité, nul si l'emploi familial n'a aucun lien statistique avec l'activité mesurable du parlementaire, et négatif si l'emploi familial est corrélé à une moindre activité. Le coefficient $\beta_{2}$ estime quant à lui le lien entre le nombre total de collaborateurs d'un parlementaire et son activité mesurée au Parlement. Il s'agit d'une variable de contrôle importante pour interprêter $\beta_{1}$ car elle permet de comparer les parlementaires qui emploient ou non des membres de leur famille à nombre de collaborateurs égal. Ce lien est supposé positif, puisque les parlementaires les plus investis sont généralement ceux qui emploient le plus de collaborateurs (corrélation) et parce que les collaborateurs sont supposés assister le parlementaire dans son travail pour plus d'efficacité (causalité). Concernant $\beta_{1}$, une source possible de biais serait une erreur de mesure, puisque la méthode d'appariement des emplois familiaux repose sur la simple comparaison des noms de famille des parlementaires et de leurs collaborateurs. La variable EmploiFamilial est

\footnotetext{
${ }^{5}$ Ces choix s'expliquent à nouveau par la forme de la distribution des indicateurs d'activité 3 à 12 , très étirée vers la droite. Pour ces 10 variables, on calcule leur logarithme comme Activite $=\log (Y+1)$. Cette spécification log-linéaire du modèle permet d'éviter que les valeurs extrêmes ne surdéterminent les coefficients estimés.
} 
donc une mesure imparfaite de la réalité des emplois familiaux au Parlement. Ces erreurs de mesure auront donc tendance à réduire le lien statistique entre EmploiFamilial et Activite, c'est-à-dire à rapprocher $\beta_{1}$ de zéro. Il faut donc prendre $\beta_{1}$ comme une estimation conservatrice de la réalité des différences d'activité entre parlementaires employant ou non un membre de leur famille.

\subsection{Résultats}

Les résultats de l'estimation de l'Equation 1 par la méthode des Moindres Carrés Ordinaires apparaissent dans les Tables 6 et 7 .

En nous concentrant tout d'abord sur les résultats pour le score global d'activité (Table 6), nous remarquons que les parlementaires qui emploient un membre de leur famille ont, toutes chose égales par ailleurs, un score d'activité significativement plus faible que les autres. Ce résultat, comme tous ceux qui suivent, est robuste à l'inclusion d'effets fixes pour chaque groupe parlementaire de l'Assemblée et du Sénat, et même d'effets fixes par département d'élection. Le coefficient associé à la variable $N b C o l l a b$ est à l'inverse significatif et positif, et suggère que le score d'activité des parlementaires croit avec le nombre de collaborateurs qu'ils embauchent. Il n'existe pas de différence significative dans le score des hommes et des femmes, mais des différences en fonction de l'age et du cumul des mandats. Enfin, les députés et les parlementaires des DOM-TOM enregistrent des scores d'activité significativement plus faibles que les autres.

Ces résultats, obtenus à partir d'un score d'activité construit par ACP, méritent cependant d'être complétés puisque ce score unique néglige une partie de la richesse des activités des parlementaires. Pour étudier l'hétérogénéité de ces résultats par type d'activité, nous étudions maintenant les estimations pour chacun des 12 indicateurs d'activité (Table 7).

Tout d'abord, on note que la variable $N b C o l l a b$ est systématiquement associée à un coefficient $\beta_{2}$ de signe positif, sauf pour la variable Ado/Sign. Ce coefficient estimé n'est pas significativement différent de zéro pour plusieurs mesures d'activités, comme le nombre total de semaines de présence au Parlement ou les présences en commissions. Cependant, le coefficient estimé est positif et significatif concernant les interventions orales dans l'Hemicycle, l'écriture et la signature de propositions de lois, ou encore les questions écrites et orales posées au gouvernement (des activités pour lesquelles les assistants parlementaires ont souvent un rôle important à jouer). Concernant les variables de contrôle, on peut noter des différences toujours significatives d'activité entre députés et sénateurs, qui vont dans un sens ou dans l'autre selon l'indicateur d'activité utilisé. On observe quelques différences en fonction du genre (les parlementaires hommes font par exemple moins souvent adopter les amendements qu'ils signent), 
de l'âge et du nombre de mandats (les parlementaires qui ne cumulent pas d'autres mandats sont particulièrement plus présents). Enfin, les élus des DOM-TOM et Français de l'étranger sont sensiblement moins présents et actifs que les autres au Parlement, ce qui peut naturellement s'expliquer par la distance géographique les séparant de Paris.

Concernant la principale variable d'intérêt, EmploiFamilial, on note d'abord que la plupart des coefficients estimés (8/12) sont de signe négatif. Parmi ceux-ci, on estime 6 coefficients qui sont significativement négatifs, ce qui suggère un lien négatif entre le fait d'employer un membre de la famille et le niveau d'activité observable du parlementaire sur l'année écoulée. En moyenne et toutes choses égales par ailleurs, on estime ainsi que les parlementaires employant un membre de leur famille ont été moins présents de 1,2 semaines sur l'année écoulée, ont assisté à 6 réunions de commissions en moins, et sont intervenus près de $30 \%$ de moins en commissions que les autres parlementaires. Ces parlementaires rendent également sensiblement moins ( $-13 \%$ environ) de rapports parlementaires, et font adopter moins d'amendements. Les autres coefficients négatifs sont également d'une assez grande magnitude mais demeurent non-significatifs. A l'inverse, aucun des 4 coefficients estimés de signe positif n'est statistiquement différent de zéro. En moyenne et toutes choses égales par ailleurs, l'emploi familial pratiqué par les parlementaires n'est donc associé positivement à aucun type d'activité observable. Au contraire, les estimations montrent que l'embauche de collaborateurs familiaux est associée à une moindre activité des parlementaires selon plusieurs indicateurs.

\subsection{Moindre présence au Parlement ou moindre productivité ?}

A ce stade, on pourrait penser que cette moindre activité parlementaire totale sur l'année écoulée peut directement s'expliquer par la moindre présence physique de ces élus à Paris (en moyenne, moins 1,2 semaines de présence - Table 7), et témoigne simplement d'une préférence pour le travail de terrain en circonscription. Dans leur étude des députés français, les politistes Costa et Kerrouche (2007) montrent bien que plusieurs types d'élus coexistent à l'Assemblée, certains étant très attachés au travail de terrain et à la circonscription, d'autres étant plus intéressés par le travail strictement législatif et de contrôle à Paris.

Pour pouvoir conclure sur la productivité ou l'efficience des parlementaires à temps de travail donné, nous calculons pour chaque indicateur d'activité son niveau par semaine travaillée à Paris. Par exemple, pour le nombre de réunions de commissions, nous calculons la variable WComPres $=$ ComPres/Semaines pour mesurer l'investissement du parlementaire dans le travail en commissions lorsqu'il est présent à Paris. Pour tous les autres indicateurs, nous calculons $W Y=\log (1+Y /$ Semaines $)$, pour tenir à nouveau compte de leur distribtion très 
étirée vers la droite. Ces variables de productivité ne peuvent être calculés pour les 3 députés n'ayant jamais été présent sur l'année écoulée $(\mathrm{N}=893)$.

Les résultats de ces modèles de productivité apparaissent dans la Table 8. Les estimations font à nouveau apparaître de nombreux coefficient négatifs $(6 / 10)$ dont plusieurs sont statistiquement différents de zéro : ainsi, les parlementaires pratiquant l'emploi familial assistent à 0,1 réunion de commissions en moins par semaine de présence à Paris, interviennent significativement moins en commissions et dans l'hémicycle, rendent moins de rapports et font adopter moins d'amendements. Cependant, ils rédigent plus de propositions de lois par semaine de présence (les propositions sont cependant très rarement adoptées par le Parlement) et posent plus de questions écrites au gouvernement. Ces résultats montrent donc que, pris dans leur ensemble, la moindre activité des parlementaires qui emploient des membres de leur famille ne s'explique pas uniquement par leur moindre présence au Parlement (en faveur du travail en circonscription par exemple) : ils témoignent aussi d'une moindre productivité avec moins de travail parlementaire observable par unité de temps travaillé à l'Assemblée ou au Sénat. 
Table 6: Régressions par MCO de la relation entre collaborateurs, emplois familiaux et score d'activité des parlementaires

\begin{tabular}{lccc}
\hline \hline & $(1)$ & $(2)$ & $(3)$ \\
& Score & Score & Score \\
\hline Emploi familial & $-0.383^{*}$ & $-0.370^{*}$ & $-0.357^{+}$ \\
& $(0.184)$ & $(0.183)$ & $(0.191)$ \\
Nb collab & $0.184^{* *}$ & $0.211^{* *}$ & $0.243^{* * *}$ \\
& $(0.068)$ & $(0.067)$ & $(0.073)$ \\
Homme & -0.068 & -0.140 & -0.182 \\
& $(0.145)$ & $(0.146)$ & $(0.147)$ \\
Age & $-0.029^{* * *}$ & $-0.030^{* * *}$ & $-0.025^{* * *}$ \\
& $(0.007)$ & $(0.007)$ & $(0.007)$ \\
Seul mandat & $0.443^{*}$ & $0.561^{* *}$ & 0.228 \\
& $(0.199)$ & $(0.201)$ & $(0.220)$ \\
Depute & $-0.692^{* * *}$ & & \\
Dom-Tom & $(0.148)$ & & \\
Constant & $-1.196^{* * *}$ & $-1.346^{* * *}$ & \\
& $(0.247)$ & $(0.250)$ & \\
\hline Parti & $1.744^{* * *}$ & $1.778^{*}$ & -0.091 \\
Departement & $(0.486)$ & $(0.741)$ & $(1.949)$ \\
r2 & - & $\mathrm{X}$ & $\mathrm{X}$ \\
\hline \hline N $=896$. Ecart-types entre parentheses. & $\mathrm{X}$ \\
$+{ }_{p}<0.10,{ }^{*} p<0.05,{ }^{* *} p<0.01,{ }^{* * *} p<0.001$ \\
\end{tabular}


Table 7: Régressions par MCO de la relation entre collaborateurs, emplois familiaux et activités des parlementaires

\begin{tabular}{|c|c|c|c|c|c|c|c|c|c|c|c|c|}
\hline & (1) & (2) & (3) & (4) & (5) & (6) & (7) & (8) & (9) & (10) & (11) & (12) \\
\hline & Semaines & ComPres & ComInter & HemInter & Rapports & AmSign & AmAdo & Ado/Sign & PropEcrit & PropSign & QEcrites & QOrales \\
\hline \multirow[t]{2}{*}{ Emploi familial } & $-1.224^{+}$ & $-5.887^{*}$ & $-0.322^{*}$ & -0.198 & $-0.138^{*}$ & 0.020 & $-0.261^{*}$ & $-0.267^{* *}$ & 0.057 & 0.025 & 0.145 & -0.093 \\
\hline & $(0.699)$ & $(2.668)$ & $(0.146)$ & $(0.145)$ & $(0.061)$ & $(0.120)$ & $(0.104)$ & $(0.087)$ & $(0.052)$ & $(0.074)$ & $(0.111)$ & $(0.061)$ \\
\hline \multirow[t]{2}{*}{$\mathrm{Nb}$ collab } & 0.389 & 0.967 & 0.058 & $0.110^{*}$ & 0.015 & 0.069 & 0.033 & -0.037 & $0.099^{* * *}$ & $0.090^{* *}$ & $0.152^{* * *}$ & $0.043^{+}$ \\
\hline & $(0.259)$ & $(0.990)$ & $(0.054)$ & $(0.054)$ & $(0.023)$ & $(0.045)$ & $(0.039)$ & $(0.032)$ & $(0.019)$ & $(0.027)$ & $(0.041)$ & $(0.023)$ \\
\hline \multirow[t]{2}{*}{ Homme } & 0.172 & -0.534 & $-0.235^{*}$ & -0.091 & 0.060 & -0.067 & $-0.297^{* * *}$ & $-0.271^{* * *}$ & $0.194^{* * *}$ & 0.028 & 0.068 & -0.079 \\
\hline & $(0.555)$ & $(2.118)$ & $(0.116)$ & $(0.115)$ & $(0.049)$ & $(0.095)$ & $(0.082)$ & $(0.068)$ & $(0.042)$ & $(0.058)$ & $(0.088)$ & $(0.049)$ \\
\hline \multirow[t]{2}{*}{ Age } & -0.036 & 0.039 & -0.004 & $-0.036^{* * *}$ & -0.002 & $-0.009^{+}$ & $-0.007^{+}$ & -0.001 & $-0.007^{* * *}$ & 0.002 & $-0.016^{* * *}$ & $-0.010^{* * *}$ \\
\hline & $(0.026)$ & $(0.098)$ & $(0.005)$ & $(0.005)$ & $(0.002)$ & $(0.004)$ & $(0.004)$ & $(0.003)$ & $(0.002)$ & $(0.003)$ & $(0.004)$ & $(0.002)$ \\
\hline \multirow[t]{2}{*}{ Seul mandat } & $2.679^{* * *}$ & $11.458^{* * *}$ & $0.330^{*}$ & $0.303^{+}$ & 0.094 & -0.146 & -0.143 & 0.000 & 0.072 & -0.121 & $-0.221^{+}$ & 0.011 \\
\hline & $(0.761)$ & $(2.904)$ & $(0.159)$ & $(0.158)$ & $(0.067)$ & $(0.131)$ & $(0.113)$ & $(0.094)$ & $(0.057)$ & $(0.080)$ & $(0.121)$ & $(0.067)$ \\
\hline \multirow[t]{2}{*}{ Dom-Tom } & $-11.443^{* * *}$ & $-22.570^{* * *}$ & $-0.444^{*}$ & $-0.359^{+}$ & -0.104 & 0.134 & $0.254^{+}$ & 0.132 & -0.067 & $-0.434^{* * *}$ & $-0.692^{* * *}$ & $-0.195^{*}$ \\
\hline & (0.937) & $(3.580)$ & $(0.195)$ & $(0.194)$ & $(0.082)$ & $(0.161)$ & $(0.139)$ & $(0.117)$ & $(0.070)$ & $(0.099)$ & $(0.150)$ & $(0.082)$ \\
\hline \multirow[t]{2}{*}{ Depute } & $-4.992^{* * *}$ & $-28.570^{* * *}$ & $-0.304^{* *}$ & $-0.696^{* * *}$ & $-0.267^{* * *}$ & $1.044^{* * *}$ & $0.427^{* * *}$ & $-0.604^{* * *}$ & $-0.153^{* * *}$ & $0.953^{* * *}$ & 0.111 & $0.224^{* * *}$ \\
\hline & $(0.563)$ & $(2.151)$ & & $(0.117)$ & $(0.050)$ & $(0.097)$ & & & $(0.042)$ & $(0.059)$ & $(0.090)$ & $(0.049)$ \\
\hline \multirow[t]{2}{*}{ Constant } & $32.661^{* * *}$ & $66.333^{* * *}$ & $3.655^{* * *}$ & $5.534^{* * *}$ & $0.845^{* * *}$ & $4.998^{* * *}$ & $3.804^{* * *}$ & $3.501^{* * *}$ & $0.362^{* *}$ & $1.573^{* * *}$ & $2.643^{* * *}$ & $1.372^{* * *}$ \\
\hline & $(1.840)$ & $(7.027)$ & $(0.383)$ & $(0.381)$ & $(0.162)$ & $(0.316)$ & & $(0.230)$ & & $(0.194)$ & $(0.294)$ & $(0.161)$ \\
\hline $\mathrm{r} 2$ & 0.209 & 0.231 & 0.030 & 0.083 & 0.045 & 0.177 & 0.075 & 0.143 & 0.063 & 0.335 & 0.087 & 0.093 \\
\hline
\end{tabular}

$\mathrm{N}=896$. Ecart-types entre parentheses. Les colonnes (1) et (2) correspondent à des régressions linéaires, les colonnes (3)-(12) à des régressions log-linéaires.

$+p<0.10,{ }^{*} p<0.05,{ }^{* *} p<0.01,{ }^{* * *} p<0.001$ 
Table 8: Régressions par MCO des activités des parlementaires par semaine de présence

\begin{tabular}{|c|c|c|c|c|c|c|c|c|c|c|}
\hline & (1) & (2) & (3) & (4) & (5) & (6) & (7) & (8) & (9) & (10) \\
\hline & WComPres & WComInter & WHemInter & WRapports & WAmSign & WAmAdo & WPropEcrit & WPropSign & WQEcrit & WQOral \\
\hline \multirow[t]{2}{*}{ Emploi familial } & $-0.102^{+}$ & $-0.158^{*}$ & $-0.119^{+}$ & $-0.010^{+}$ & 0.060 & $-0.112^{*}$ & $0.009^{+}$ & 0.033 & $0.066^{+}$ & -0.008 \\
\hline & $(0.061)$ & $(0.063)$ & $(0.069)$ & $(0.006)$ & $(0.087)$ & $(0.050)$ & $(0.005)$ & $(0.030)$ & $(0.034)$ & $(0.007)$ \\
\hline \multirow[t]{2}{*}{$\mathrm{Nb}$ collab } & 0.020 & 0.030 & $0.052^{*}$ & 0.001 & 0.049 & 0.013 & $0.009^{* * *}$ & $0.035^{* *}$ & $0.044^{* * *}$ & 0.003 \\
\hline & $(0.023)$ & $(0.023)$ & $(0.026)$ & $(0.002)$ & $(0.032)$ & $(0.018)$ & $(0.002)$ & $(0.011)$ & $(0.013)$ & $(0.003)$ \\
\hline \multirow[t]{2}{*}{ Homme } & -0.058 & $-0.099^{*}$ & -0.015 & 0.003 & -0.010 & $-0.167^{* * *}$ & $0.014^{* * *}$ & 0.031 & 0.042 & -0.003 \\
\hline & $(0.049)$ & $(0.050)$ & $(0.055)$ & $(0.004)$ & $(0.069)$ & $(0.039)$ & $(0.004)$ & $(0.023)$ & $(0.027)$ & $(0.005)$ \\
\hline \multirow[t]{2}{*}{ Age } & $0.004^{+}$ & -0.001 & $-0.014^{* * *}$ & -0.000 & -0.004 & -0.003 & $-0.000^{*}$ & $0.002^{*}$ & $-0.003^{*}$ & $-0.001^{* *}$ \\
\hline & $(0.002)$ & $(0.002)$ & $(0.003)$ & $(0.000)$ & $(0.003)$ & $(0.002)$ & $(0.000)$ & $(0.001)$ & $(0.001)$ & $(0.000)$ \\
\hline \multirow[t]{2}{*}{ Seul mandat } & $0.214^{* *}$ & 0.095 & $0.125^{+}$ & 0.004 & $-0.227^{*}$ & $-0.123^{*}$ & 0.003 & $-0.061^{+}$ & $-0.092^{*}$ & -0.011 \\
\hline & $(0.067)$ & $(0.068)$ & $(0.075)$ & $(0.006)$ & $(0.095)$ & $(0.054)$ & $(0.006)$ & $(0.032)$ & $(0.037)$ & $(0.007)$ \\
\hline \multirow[t]{2}{*}{ Dom-Tom } & $-0.145^{+}$ & 0.048 & 0.092 & 0.010 & $0.621^{* * *}$ & $0.518^{* * *}$ & 0.004 & 0.008 & -0.058 & $0.038^{* * *}$ \\
\hline & $(0.083)$ & $(0.085)$ & (0.094) & (0.008) & $(0.117)$ & $(0.067)$ & $(0.007)$ & $(0.040)$ & $(0.046)$ & $(0.009)$ \\
\hline \multirow[t]{2}{*}{ Depute } & $-0.686^{* * *}$ & -0.029 & $-0.212^{* * *}$ & $-0.017^{* * *}$ & $1.057^{* * *}$ & $0.323^{* * *}$ & $-0.007^{+}$ & $0.359^{* * *}$ & $0.081^{* *}$ & $0.044^{* * *}$ \\
\hline & $(0.049)$ & $(0.050)$ & $(0.056)$ & $(0.005)$ & $(0.070)$ & $(0.040)$ & $(0.004)$ & $(0.024)$ & $(0.027)$ & $(0.006)$ \\
\hline \multirow[t]{2}{*}{ Constant } & $1.932^{* * *}$ & $0.860^{* * *}$ & $1.643^{* * *}$ & $0.055^{* * *}$ & $1.702^{* * *}$ & $0.959^{* * *}$ & 0.014 & -0.039 & $0.327^{* * *}$ & $0.081^{* * *}$ \\
\hline & $(0.162)$ & $(0.165)$ & $(0.183)$ & $(0.015)$ & $(0.229)$ & $(0.131)$ & $(0.014)$ & $(0.078)$ & $(0.090)$ & $(0.018)$ \\
\hline $\mathrm{r} 2$ & 0.234 & 0.019 & 0.053 & 0.026 & 0.289 & 0.164 & 0.049 & 0.292 & 0.071 & 0.127 \\
\hline
\end{tabular}

$\mathrm{N}=$ 893. Ecart-types entre parentheses. La colonne (1) correspond à une régression linéaire, les colonnes (2)-(10) à des régressions log-linéaires .

$+p<0.10,{ }^{*} p<0.05,{ }^{* *} p<0.01,{ }^{* * *} p<0.001$ 


\subsection{Effet de sélection ou effet causal ?}

Comme expliqué dans la section 4.1, ces liens négatifs entre emploi familial et activité parlementaire n'impliquent pas nécessairement la présence d'un effet causal négatif (les collaborateurs familiaux provoquent une réduction de l'activité des parlementaires), mais peuvent également illustrer un simple effet de sélection (les parlementaires qui emploient des membres de leur famille sont, en moyenne et toutes choses égales par ailleurs, les moins actifs au Parlement). Pour tenter d'évaluer empiriquement la validité de ces deux hypothèses, nous avons recours à une estimation par instrumentation. Cette méthode vise à corriger le biais de sélection provenant d'une éventuelle corrélation entre l'emploi de collaborateurs familiaux et les déterminants inobservables de l'activité. On peut par exemple penser que la nature des motivations des parlementaires (service de l'intérêt général, extraction de rentes, ou autres) est une variable inobservable qui peut expliquer à la fois le choix de privilégier ou non un membre de sa famille, et l'effort fourni par le parlementaire durant son mandat.

Pour tenter de corriger ce biais de sélection sur les inobservables, il nous faut exploiter une variable fortement corrélée à la pratique de l'emploi familial mais non-corrélée a priori aux déterminants inobservables de l'activité. A défaut de règle stricte qui contraindrait l'emploi familial pour certains parlementaires et pas pour d'autres (sur des critères arbitraires, ou exogènes), nous retenons comme variable instrumentale la situation matrimoniale des parlementaires. Pour chaque député et sénateur, cette variable dichotomique indique si le parlementaire est en couple ou si, à l'inverse, aucun conjoint n'est connu publiquement (sources officielles, presse). D'après les données compilées par la plateforme Projet Arcadie, $62 \%$ des 920 parlementaires vivent ainsi en couple de manière connue. Cette variable représente un instrument potentiellement crédible car, d'une part, elle est corrélée à la présence de collaborateurs familiaux dans l'équipe d'un parlementaire, et d'autre part, elle ne semble a priori pas directement corrélée aux déterminants inobservables de l'activité (comme la nature des motivations des parlementaires). Bien qu'une vaste littérature s'intéresse aux liens existants entre situation familiale et offre de travail, on pense en effet que les parlementaires ne sont pas des travailleurs comme les autres : ils touchent une indemnité fixe, et non un salaire qui varierait en fonction de leur effort ou d'heures supplémentaires; ils sont souvent passionnés par leur mandat et ne font donc pas le même arbitrage "travail-loisir"; ils sont souvent âgés, sans enfant à charge. Tous ces élements tendent à soutenir la validité de notre instument dans ce contexte. 
Le modèle estimé par Moindres Carrés en Deux Etapes (MC2E) est le suivant :

$$
\begin{aligned}
\text { EmploiFamilial }_{i} & =\alpha_{0}+\alpha_{1} \text { EnCouple }_{i}+\alpha_{2} \text { NbCollab }_{i}+\gamma^{\prime} X_{i}+e_{i} \\
\text { Activite }_{i} & =\beta_{0}+\beta_{1} \text { EmploiFamilial }_{i}+\beta_{2} \text { NbCollab }_{i}+\theta^{\prime} X_{i}+u_{i}
\end{aligned}
$$

L'estimation de la régression instrumentale (équation 2) montre comme attendu une forte relation entre la situation matrimoniale des parlementaires et leur recours aux emplois familiaux $\left(\hat{\alpha}_{1}=0,069\right.$; écart-type $\left.=0,024\right)$. Toutes choses égales par ailleurs, les parlementaires en couple ont une probabilité d'employer un collaborateur familial de 7 points supérieure à celles des autres parlementaires, soit une hausse d'environ 50\% (taux d'emploi familial de $14 \%$ dans l'échantillon). Cette différence est significative au seuil de $1 \%$ et la statistique de Fisher est proche du seuil de $10($ Fstat $=8,2)$. Ces résultats sont disponibles dans la Table 11 en annexe. L'inclusion d'effets fixes par parti fournit des estimations similaires.

Les résultats de l'estimation de l'équation d'intérêt (équation 3) par MC2E apparaissent dans la Table 9. Contrairement aux résultats de la section 4.2, aucun des coefficients associés à la variable d'emploi familial n'est désormais significatif. La plupart des coefficients sont positifs, mais aucun n'est estimé avec précision. A l'inverse, le rôle des autres variables explicatives sur l'activité des parlementaires évolue peu par rapport à la Table 7. Ces estimations instrumentales suggèrent donc qu'il n'existe pas d'effet causal significatif de l'emploi familial dans l'activité observable des députés et sénateurs au Parlement. On observe la même absence d'effet causal significatif en régressant les variables de productivité, où les indicateurs d'activité sont pondérés par le temps de présence au Parlement (Table 10), ou le score d'activité issu de notre ACP.

La variable endogène EmploiFamilial étant dichotomique, nous savons que l'estimateur utilisé (MC2E) est convergent mais pas efficace. La méthode en trois étapes proposée par Wooldridge 2010,939-940) permet d'améliorer l'efficacité de l'instrumentation en tenant compte de la nature dichomotique de l'endogène (par une première étape en probit). Nous appliquons cette méthode sur nos données, avec le même instrument. Les résultats, disponibles sur demande, sont qualitativement similaires aux précédents puisque les coefficents associés à l'emploi familial demeurent non-significatifs et de signes variables.

Ces résultats nuls accréditent plutôt l'hypothèse selon laquelle les écarts d'activité et de productivité estimés initialement (Tables 6, 7 et 8) témoignent avant tout d'un simple effet de sélection : les parlementaires qui emploient des membres de leur famille parmi leurs collaborateurs sont par ailleurs des élus moins présents et moins actifs que les autres au Parlement. Nous ne trouvons à l'inverse pas de signe d'un effet causal clair de l'emploi de membres de la famille (au détriment d'autres collaborateurs) sur l'activité des parlementaires français. 
Table 9: Régressions par Moindres Carres en 2 étapes

\begin{tabular}{|c|c|c|c|c|c|c|c|c|c|c|c|c|}
\hline & (1) & (2) & (3) & (4) & (5) & (6) & (7) & (8) & (9) & (10) & (11) & $(12)$ \\
\hline & Semaines & ComPres & ComInter & HemInter & Rapports & AmSign & AmAdo & Ado/Sig & PropEcrit & PropSign & QEcrites & QOrales \\
\hline \multirow[t]{2}{*}{ Emploi familial } & 4.178 & 11.449 & 1.633 & 1.423 & 0.530 & 0.331 & 0.164 & -0.078 & 0.100 & 0.101 & -1.751 & -0.416 \\
\hline & $(7.508)$ & $(28.400)$ & $(1.661)$ & (1.608) & $(0.680)$ & $(1.254)$ & $(1.090)$ & $(0.970)$ & $(0.544)$ & $(0.767)$ & $(1.335)$ & $(0.646)$ \\
\hline \multirow[t]{2}{*}{$\mathrm{Nb}$ collab } & 0.210 & 0.394 & -0.007 & 0.056 & -0.007 & 0.059 & 0.019 & -0.043 & $0.098^{* * *}$ & $0.088^{*}$ & $0.215^{* * *}$ & $0.053^{+}$ \\
\hline & $(0.363)$ & $(1.375)$ & $(0.080)$ & $(0.078)$ & $(0.033)$ & $(0.061)$ & $(0.053)$ & $(0.044)$ & $(0.026)$ & $(0.037)$ & $(0.065)$ & $(0.031)$ \\
\hline \multirow[t]{2}{*}{ Homme } & -0.043 & -1.225 & $-0.313^{*}$ & -0.155 & 0.033 & -0.079 & $-0.314^{* * *}$ & $-0.279^{* * *}$ & $0.193^{* * *}$ & 0.025 & 0.143 & -0.066 \\
\hline & $(0.644)$ & $(2.434)$ & $(0.142)$ & $(0.138)$ & $(0.058)$ & $(0.107)$ & $(0.093)$ & $(0.080)$ & $(0.047)$ & $(0.066)$ & $(0.114)$ & $(0.055)$ \\
\hline \multirow[t]{2}{*}{ Age } & -0.065 & -0.053 & -0.015 & $-0.044^{* * *}$ & -0.006 & -0.010 & -0.009 & -0.002 & $-0.007^{*}$ & 0.002 & -0.006 & $-0.008^{+}$ \\
\hline & $(0.048)$ & $(0.181)$ & $(0.011)$ & $(0.010)$ & $(0.004)$ & $(0.008)$ & $(0.007)$ & $(0.006)$ & $(0.003)$ & $(0.005)$ & $(0.009)$ & $(0.004)$ \\
\hline \multirow[t]{2}{*}{ Seul mandat } & $3.078^{* *}$ & $12.736^{* * *}$ & $0.474^{*}$ & $0.422^{*}$ & $0.144^{+}$ & -0.123 & -0.112 & 0.014 & 0.075 & -0.116 & $-0.360^{*}$ & -0.013 \\
\hline & $(0.957)$ & $(3.620)$ & $(0.212)$ & $(0.205)$ & $(0.087)$ & $(0.160)$ & $(0.139)$ & $(0.119)$ & (0.069) & (0.098) & $(0.170)$ & $(0.082)$ \\
\hline \multirow[t]{2}{*}{ Dom-Tom } & $-12.099^{* * *}$ & $-24.674^{* * *}$ & $-0.681^{*}$ & $-0.556^{+}$ & -0.185 & 0.096 & 0.203 & 0.110 & -0.072 & $-0.443^{* *}$ & $-0.462^{*}$ & -0.155 \\
\hline & (1.324) & $(5.007)$ & $(0.293)$ & $(0.284)$ & $(0.120)$ & $(0.221)$ & $(0.192)$ & $(0.163)$ & (0.096) & $(0.135)$ & $(0.235)$ & (0.114) \\
\hline \multirow[t]{2}{*}{ Depute } & $-5.120^{* * *}$ & $-28.979^{* * *}$ & $-0.350^{* *}$ & $-0.734^{* * *}$ & $-0.283^{* * *}$ & $1.036^{* * *}$ & $0.417^{* * *}$ & $-0.608^{* * *}$ & $-0.154^{* * *}$ & $0.952^{* * *}$ & 0.155 & $0.232^{* * *}$ \\
\hline & $(0.606)$ & $(2.291)$ & $(0.134)$ & $(0.130)$ & $(0.055)$ & $(0.101)$ & & & $(0.044)$ & $(0.062)$ & $(0.108)$ & $(0.052)$ \\
\hline \multirow[t]{2}{*}{ Constant } & $34.483^{* * *}$ & $72.180^{* * *}$ & $4.315^{* * *}$ & $6.080^{* * *}$ & $1.071^{* * *}$ & $5.103^{* * *}$ & $3.947^{* * *}$ & $3.568^{* * *}$ & $0.377^{+}$ & $1.599^{* * *}$ & $2.004^{* * *}$ & $1.263^{* * *}$ \\
\hline & $(3.152)$ & $(11.924)$ & $(0.697)$ & $(0.675)$ & $(0.286)$ & $(0.527)$ & & $(0.411)$ & $(0.229)$ & $(0.322)$ & $(0.560)$ & $(0.271)$ \\
\hline r2_a & 0.149 & 0.188 & -0.176 & -0.054 & -0.090 & 0.164 & 0.050 & 0.132 & 0.055 & 0.329 & -0.220 & 0.057 \\
\hline
\end{tabular}

$\mathrm{N}=896$. Ecart-types entre parentheses. Les colonnes (1)-(2) correspondent à des régressions linéaires, les colonnes (3)-(12) à des régressions log-linéaires

$+p<0.10,{ }^{*} p<0.05,{ }^{* *} p<0.01,{ }^{* * *} p<0.001$ 
Table 10: Régressions par Moindres Carres en 2 étapes

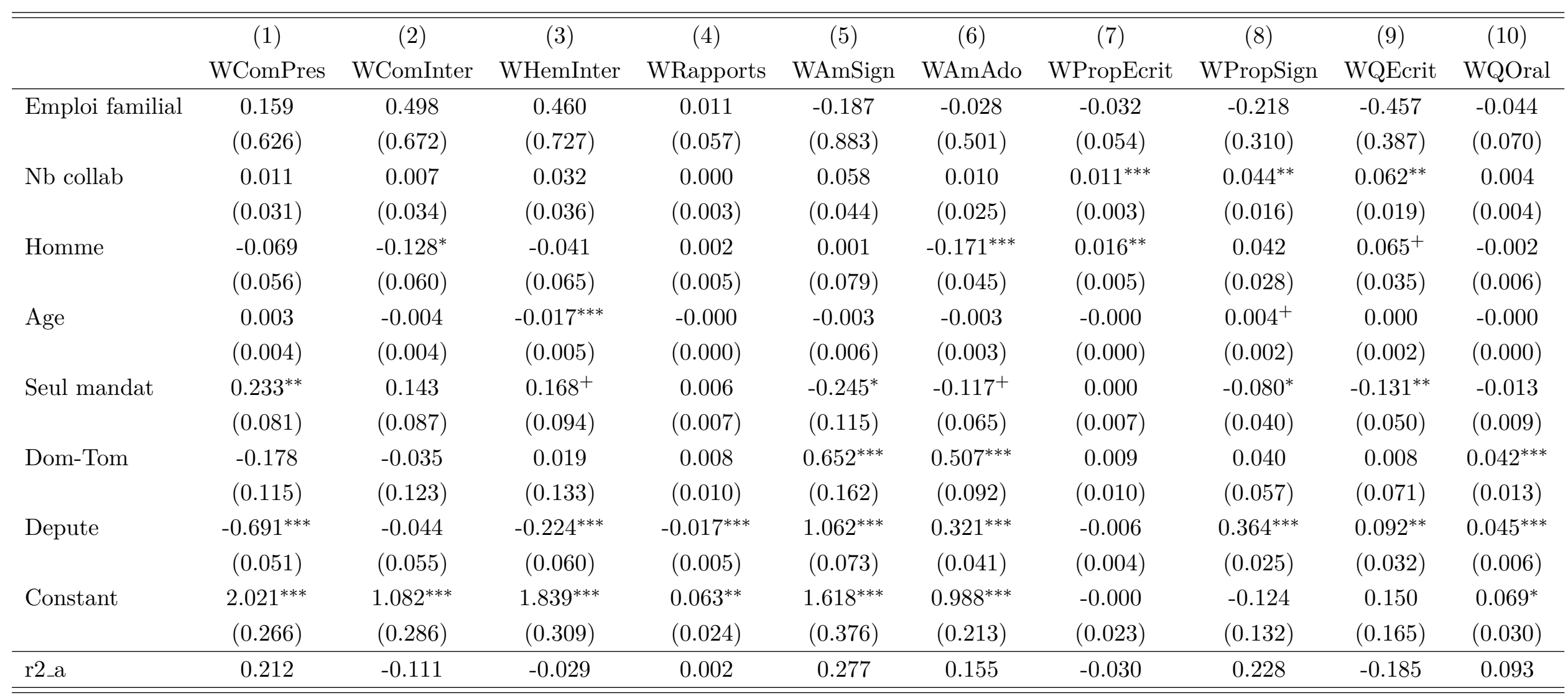

$\mathrm{N}=893$. Ecart-types entre parentheses. La colonne (1) correspond à une régression linéaire, les colonnes (2)-(10) à des régressions log-linéaires

$+p<0.10,{ }^{*} p<0.05,{ }^{* *} p<0.01,{ }^{* * *} p<0.001$ 


\section{Conclusion}

Cet article profite de la récente publication des listes de tous les collaborateurs parlementaires par l'Assemblée Nationale et le Sénat pour répondre à trois questions : (i) Combien de parlementaires emploient aujourd'hui des collaborateurs de leur famille ? (ii) Quelles caractéristiques démographiques et politiques distinguent principalement les parlementaires ayant recours à des emplois familaux des autres parlementaires? (iii) Quelle est la relation statistique entre nombre de collaborateurs, emplois familiaux, et niveau d'activité observable des parlementaires ? Cette relation est-elle causale ou illustre-t-elle un effet de sélection?

En comparant les noms de famille des 920 parlementaires actuels et de leurs 3027 collaborateurs déclarés, cette étude estime à 129 (14\%) le nombre de parlementaires employant au moins un membre de leur famille au 21 février 2017. Ce chiffre est une estimation basse de la réalité du phénomène, puisque ne sont pas détectées les épouses utilisant uniquement leur nom de jeune fille par exemple. Les autres données disponibles (enquête Medipart de 2014, opération \#TransparenceAN du Monde début 2017) suggèrent qu'environ $80 \%$ des emplois familiaux sont tout de même détectés par notre méthode d'appariemment sur les noms de famille.

En rapprochant ces données des caractéristiques des parlementaires, nous montrons que la propension à employer un membre de sa famille varie sensiblement entre parlementaires hommes $(16 \%)$ et parlementaires femmes (9\%), et en fonction de l'âge, notamment. La part des femmes de moins de 60 ans employant un membre de leur famille est ainsi de $6,5 \%$, contre $20 \%$ chez les hommes de plus de 60 ans.

Enfin, en estimant à l'aide de régressions économétriques les liens entre nombre de collaborateurs, emplois familiaux et de nombreux indicateurs d'activité parlementaire, cette étude montre plusieurs liens négatifs et significatifs (et aucun lien significativement positif) entre le fait d'employer un membre de sa famille et l'activité des élus au Parlement, en moyenne et toutes choses égales par ailleurs. De même, à temps de présence égal au Parlement, les élus qui emploient des membres de leur famille sont significativement moins productifs sur plusieurs indicateurs. Ces résultats suggèrent soit que l'emploi de collaborateurs familiaux réduit l'activité des parlementaires (effet causal), soit que les parlementaires qui emploient un membre de leur famille sont aussi des parlementaires moins actifs que les autres au Parlement (effet de sélection). Nos régressions instrumentales, exploitant la situation matrimoniale des parlementaires, supportent plutôt la deuxième hypothèse puisqu'elles ne permettent pas d'établir le moindre effet causal significatif de l'emploi familial sur les différents indicateurs d'activité. Il semble donc qu'en moyenne, emploi familial et activité réduite au Parlement témoignent d'une même pratique parlementaire. 


\section{Bibliography}

Altindag, D. T. et N. Mocan (2015). Mobile politicians: Opportunistic career moves and moral hazard. Technical report, National Bureau of Economic Research.

Barro, R. J. (1973). The control of politicians: An economic model. Public Choice 14(1), 19-42.

Costa, O. et E. Kerrouche (2007). Qui sont les députés français? Presses de Sciences Po.

Eggers, A. C. et J. Hainmueller (2009). Mps for sale? returns to office in postwar british politics. American Political Science Review 103(4), 513-533.

François, A. et L. Weill (2014). Le cumul de mandats locaux affecte-t-il l'activité des députés français ? Revue Economique 65(6), 881-906.

Gagliarducci, S. et M. Manacorda (2016). Politics in the Family : Nepotism and the Hiring Decisions of Italian Firms. Technical report, EIEF Working Paper.

Gagliarducci, S., T. Nannicini, et P. Naticchioni (2010). Moonlighting politicians. Journal of Public Economics 94(9), 688-699.

Kauder, B. et N. Potrafke (2016). Supermajorities and political rent extraction. Kyklos 69(1), $65-81$.

Navarro, J., N. G. Vaillant, et F.-C. Wolff (2012). Mesurer l'efficacité des députés au sein du parlement français. Revue française de science politique 62(4), 611-636.

Wooldridge, J. (2010). Econometric Analysis of Cross Section and Panel Data. Econometric Analysis of Cross Section and Panel Data. MIT Press. 


\section{$6 \quad$ Annexes}

Figure 1: Distribution des indicateurs d'activité dans l'échantillon final
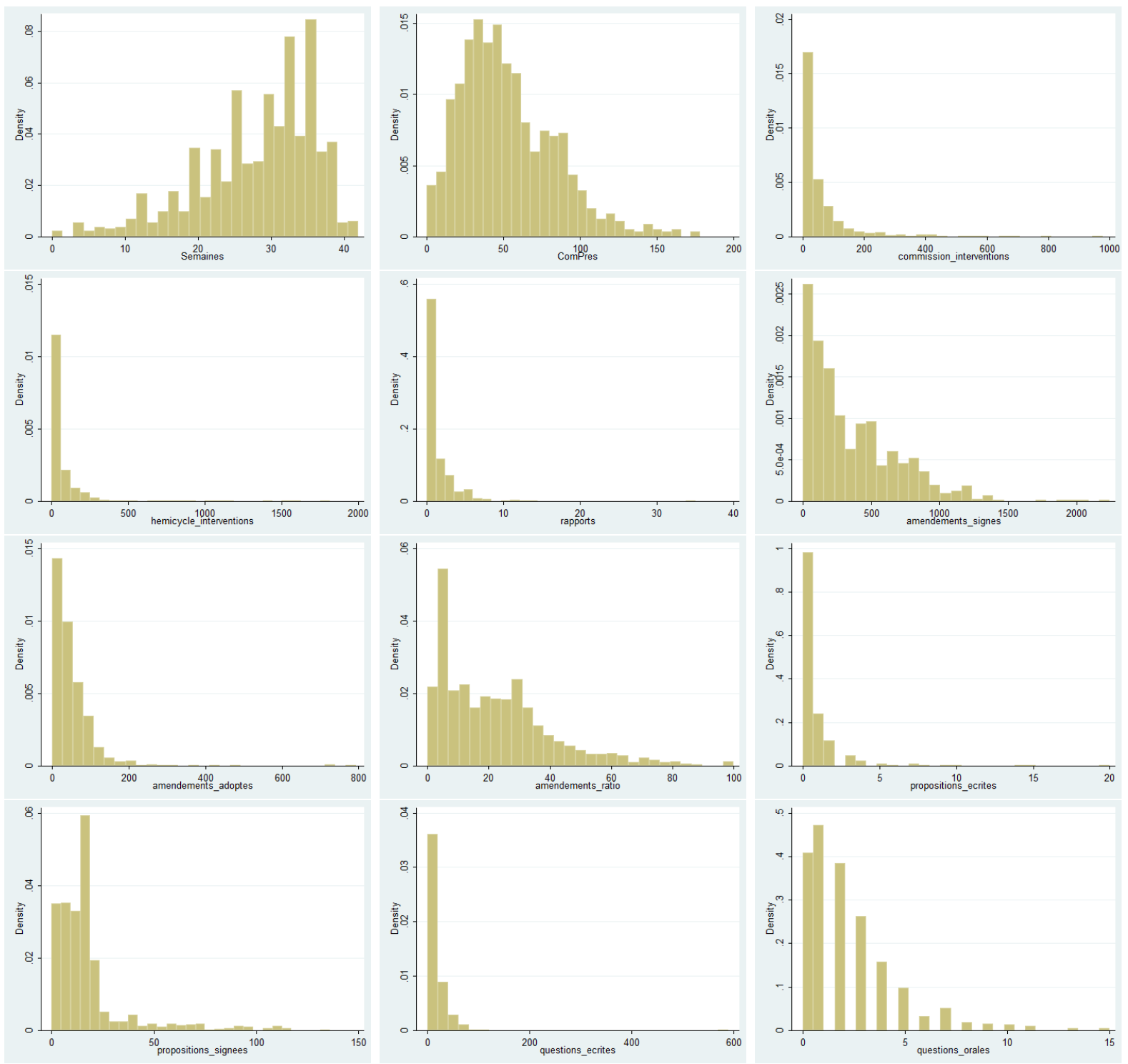
Table 11: Régression Instrumentale (1ere étape)

\begin{tabular}{|c|c|c|}
\hline & (1) & $(2)$ \\
\hline & Emploi familial & Emploi familial \\
\hline \multirow[t]{2}{*}{ VI : En couple } & $0.069^{* *}$ & $0.077^{* *}$ \\
\hline & $(0.024)$ & $(0.024)$ \\
\hline \multirow[t]{2}{*}{$\mathrm{Nb}$ collab } & $0.032^{* *}$ & $0.031^{*}$ \\
\hline & $(0.012)$ & $(0.012)$ \\
\hline \multirow[t]{2}{*}{ Homme } & 0.036 & 0.017 \\
\hline & $(0.027)$ & $(0.027)$ \\
\hline \multirow[t]{2}{*}{ Age } & $0.006^{* * *}$ & $0.006^{* * *}$ \\
\hline & $(0.001)$ & $(0.001)$ \\
\hline \multirow[t]{2}{*}{ Seul mandat } & $-0.070^{+}$ & $-0.065^{+}$ \\
\hline & $(0.036)$ & $(0.037)$ \\
\hline \multirow[t]{2}{*}{ Dom-Tom } & $0.126^{* *}$ & $0.138^{* *}$ \\
\hline & $(0.045)$ & $(0.045)$ \\
\hline \multirow[t]{2}{*}{ Depute } & 0.026 & \\
\hline & $(0.027)$ & \\
\hline \multirow[t]{2}{*}{ Constant } & $-0.417^{* * *}$ & $-0.326^{*}$ \\
\hline & $(0.092)$ & $(0.162)$ \\
\hline Parti & - & $\mathrm{X}$ \\
\hline F-stat : VI $=0$ & 8.211 & 10.252 \\
\hline $\mathrm{r} 2$ & 0.051 & 0.075 \\
\hline
\end{tabular}

Ecart-types entre parentheses.

${ }^{+} p<0.10,{ }^{*} p<0.05,{ }^{* *} p<0.01,{ }^{* * *} p<0.001$ 